\title{
INTERNAL STRUCTURE AND ROTATION OF THE SUN: FIRST RESULTS FROM THE MDI DATA
}

A.G. KOSOVICHEV, J. SCHOU, P.H. SCHERRER , R.S. BOGART, R.I. BUSH, J.T. HOEKSEMA, J. ALOISE, L. BACON,

A. BURNETTE, C. DE FOREST, P.M. GILES , K. LEIBRAND, R. NIGAM, M. RUBIN, K. SCOTT AND S.D. WILLIAMS

W.W. Hansen Experimental Physics Laboratory, Stanford University, Stanford, CA 94305, USA

SARBANI BASU AND J. CHRISTENSEN-DALSGAARD Theoretical Astrophysics Center, Danish National Research Foundation, and Institute of Physics and Astronomy, Aarhus University, DK-8000 Aarhus C, Denmark W. DÄPPEN AND E.J. RHODES, JR.

Department of Physics and Astronomy, University of Southern California, Los Angeles, CA 90089, USA T.L. DUVALL, JR.

Laboratory for Astronomy and Solar Physics, NASA Goddard Space Flight Center, Greenbelt, MD 20771, USA

R. HOWE AND M.J. THOMPSON

Astronomy Unit, Queen Mary and Westfield College, London, E1 $4 N S, U K$

D.O. GOUGH AND T. SEKII

Institute of Astronomy, and Department of Applied Mathematics and Theoretical Physics, Madingley Road, Cambridge, CB3 $O H A, U K$

J. TOOMRE

JILA, University of Colorado, CO 80309, USA

T.D. TARBELL, A.M. TITLE, D. MATHUR, M. MORRISON,

J.L.R. SABA， C.J. WOLFSON AND I. ZAYER

Lockheed-Martin Advanced Technology Center, 91-30/252, 3251

Hanover St., Palo Alto, CA 94304.

P.N. MILFORD

Parallel Rules, Inc. 41 Manzanita Ave., Los Gatos, CA 95030 
Abstract. The Medium-l Program of the Michelson Doppler Imager (MDI) instrument on board SOHO provides continuous observations of oscillation modes of angular degree, $l$, from 0 to $\sim 300$. The initial results show that the noise in the Medium- $l$ oscillation power spectrum is substantially lower than in ground-based measurements. This enables us to detect lower amplitude modes and, thus, to extend the range of measured mode frequencies. The MDI observations also reveal the asymmetry of oscillation spectral lines. The line asymmetries agree with the theory of mode excitation by acoustic sources localized in the upper convective boundary layer. The sound-speed profile inferred from the mean frequencies gives evidence for a sharp variation at the edge of the energy-generating core. In a thin layer just beneath the convection zone, helium appears to be less abundant than predicted by theory. Inverting the multiplet frequency splittings from MDI, we detect significant rotational shear in this thin layer.

\section{MDI Medium- $l$ Program}

ats

MDI has three basic helioseismology programs: Medium- $l$, Low- $l$, and Dynamics (Scherrer et al., 1996). The Medium-l data are spatial averages of the full-disk Doppler velocity out to $90 \%$ of the disk's radius measured each minute. This results in 26,000 bins of approximately 10 arcsecond resolution that provide sensitivity to solar p modes up to $l=300$. The Low- $l$ observables are velocity and continuum intensity images summed into 180 bins, with the intent of detecting oscillations up to $l=20$. The Dynamics Program provides $1024 \times 1024$ images of the whole disk, thus covering all of the $\mathrm{p}$ modes up to $l=1500$. However, the Dynamics Program can run continuously for only 2 months each year when the high-rate telemetry channel is available.

The Medium- $l$ and Low- $l$ Programs have been run with almost no interruptions since 18 April 1996. The first Dynamics observations have been carried out from 23 May to 24 July, 1996. In this paper, we present first results from the Medium- $l$ Program.

To optimize the Medium- $l$ observing program, we performed simulations of several vector-weighted binning schemes, using a 20-hour series of full-disk Dopplergrams obtained on 25 January 1996. An optimal set of Gaussian weights has been found that substantially reduces spatial aliasing in the angular degree range from 0 to 300 while preserving most of the power of the modes (see Kosovichev et al., 1997). 


\section{Medium- $l$ Power Spectrum}

A standard helioseismic data analysis procedure has been applied to obtain the oscillation power spectra from the Medium- $l$ data. 60 days of data have been processed.

The $m$-averaged power spectrum, obtained from 10 days of the data is shown in Figure 1. The ridges in the diagrams correspond to modes of different radial order $n$. The lowest weak ridge is the f mode.

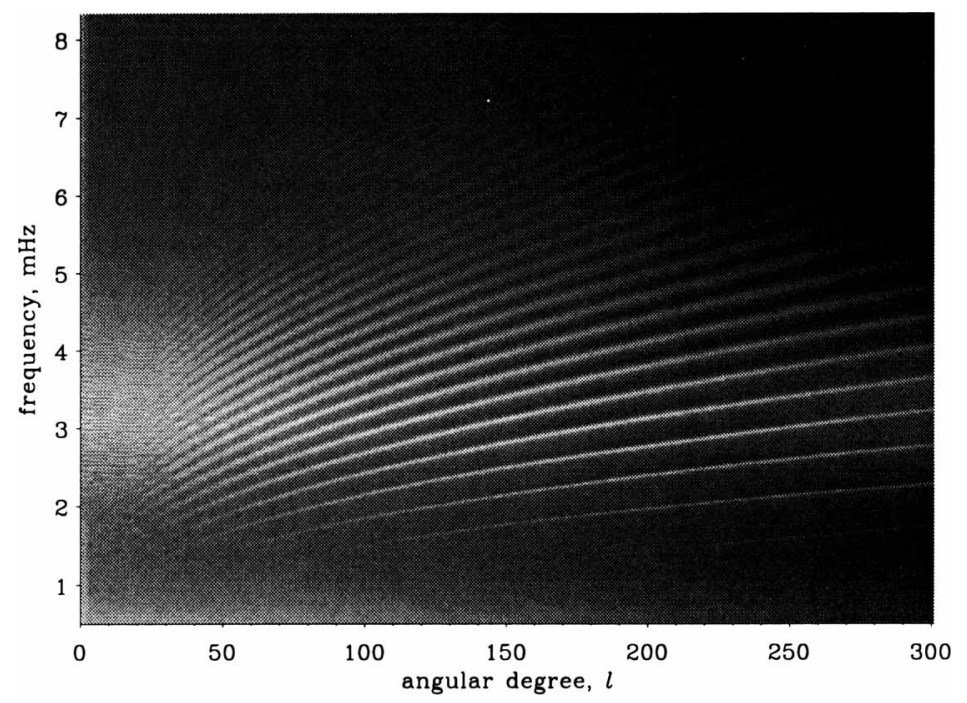

Figure 1. Power spectrum ( $l-\nu$ diagram) obtained from the MDI Medium- $l$ data for the modes averaged over $m$.

An important difference between the MDI Medium- $l$ Program and the ground-based networks is the ability to detect the low- $n$ low-frequency modes that carry substantial independent information about the solar structure and dynamics. However, the amplitude of the low- $n$ modes is very small, so long stable time series are required to detect them. One example of low-frequency modes: the f mode of $l=95$, is shown in Figure 2. Parameters of this modes have been measured for the first time. With longer time series, we should be able to observe more low-frequency modes, with even lower amplitudes. So far, the smallest mode amplitude we have been able to detect was about $1 \mathrm{~mm} / \mathrm{s}$.

The frequencies of the solar oscillations have been measured as described by Schou (1992). For the initial frequency measurements, we approximated the line profiles by Lorentzians and used a maximum likehood method to determine the parameters of the Lorentzians, taking into account possible 


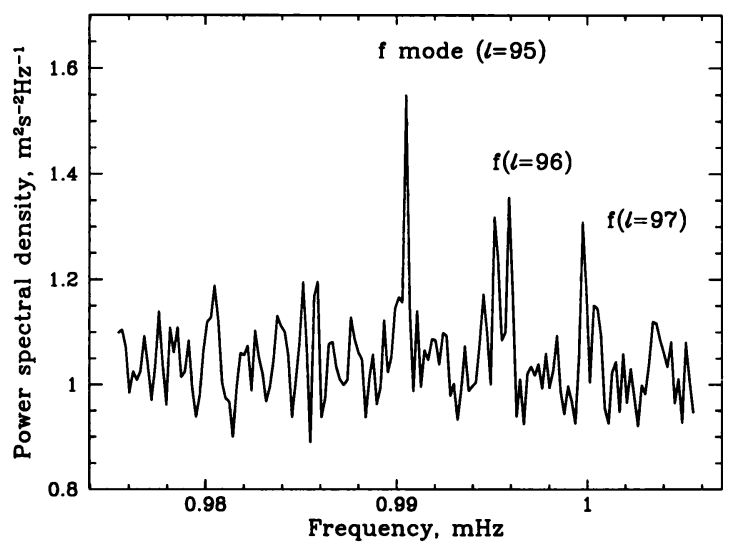

Figure 2. Power spectrum of $f$ mode of $l=95$ obtained from 2 months of the MDI data. Modes of adjacent $l$ leak into the spectrum because of imperfect spatial filtering.

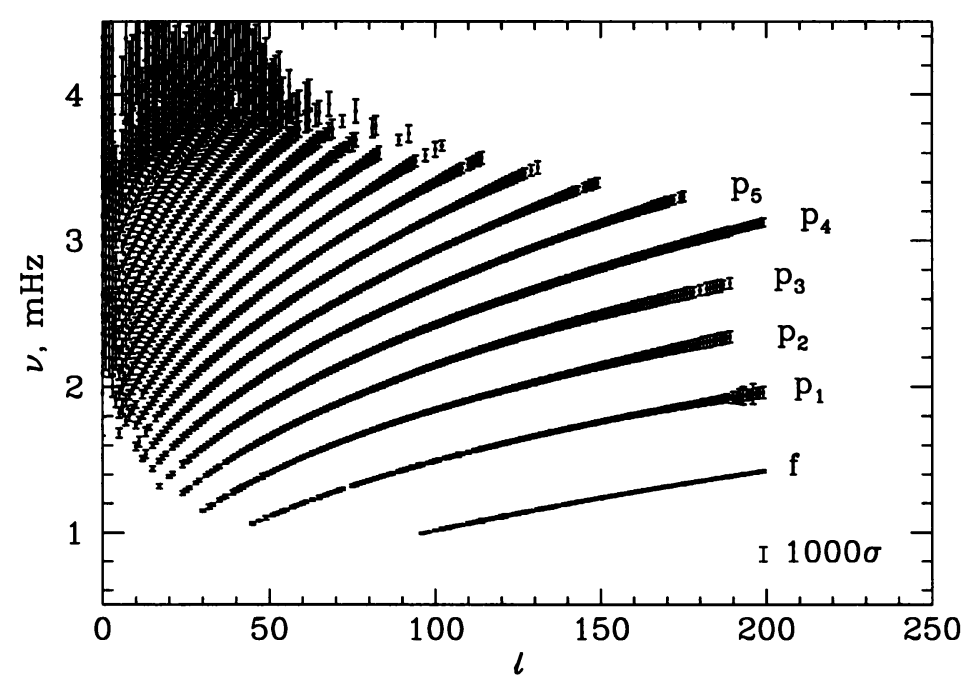

Figure 3. Mean frequencies of the mode multiplets obtained from 2 months of the Medium-l data. The error bars show the standard errors multiplied by 1000 .

overlaps of lines. The mean frequencies of mode multiplets are shown in Figure 3. The error bars in this figure indicate estimated errors multiplied by 1000 . 


\section{Line Asymmetry}

Because of the significant reduction in the noise, the Medium- $l$ data have revealed interesting characteristics of the line profiles of the oscillation power spectra. Figure 4 shows the power spectra of the modes for $l=200$.

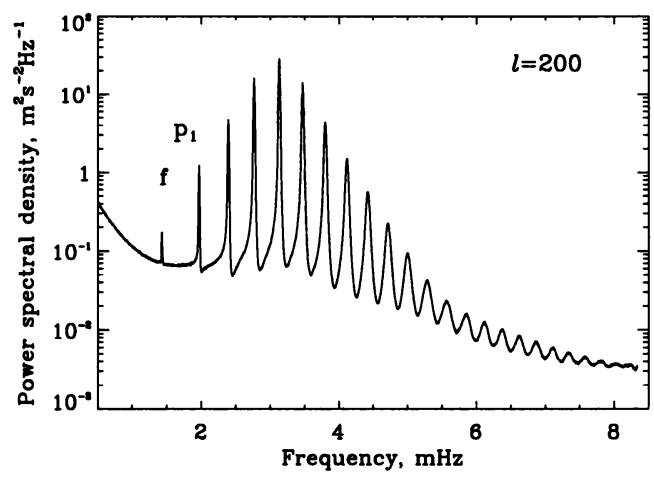

Figure 4. Power spectrum of $l=200$ modes obtained from the MDI medium- $l$ data.

The most interesting feature is the asymmetry of the line profiles. Though the asymmetry has been noticed in the ground-based data (Duvall et al., 1993), its properties have not reliably measured. Several authors have studied this problem theoretically and have found that there is an inherent asymmetry whenever the waves are excited by a localized source (e.g. Gabriel, 1992; Kumar et al. 1994).

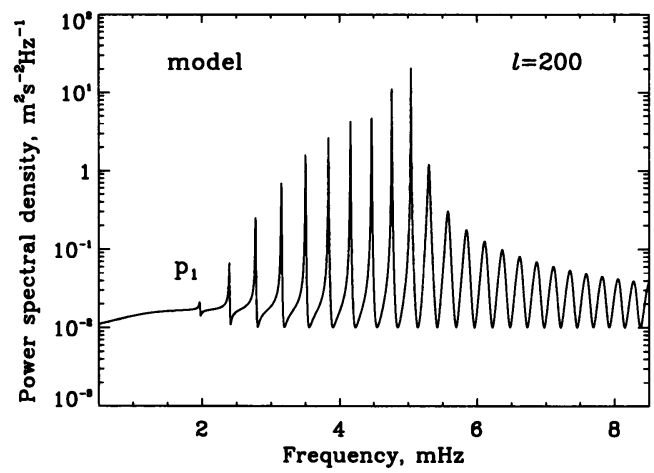

Figure 5. Theoretical power spectrum of $l=200$ modes for a model with a source of the uniform spectral density localized $80 \mathrm{~km}$ beneath the photosphere. The background noise is assumed to be uniform with the power spectral density $10^{-2} \mathrm{~m}^{2} \mathrm{~s}^{-2} \mathrm{~Hz}^{-1}$. (Nigam and Kosovichev, 1996) 
Physically, the asymmetry is an effect of interference between an outward direct wave from the source and a corresponding inward wave that passes through the region of wave propagation. Figure 5 shows a theoretical power spectrum of $\mathrm{p}$ modes of $l=200$ obtained by Nigam and Kosovichev (1996). The degree of the asymmetry depends on the relative locations of the acoustic sources and the upper reflection layer of the modes. This opens the prospect of using the observations of the line profiles of solar modes to test theories of excitation of solar and stellar oscillations and of their interaction with turbulent convection.

\section{Sound-Speed Profile}

We have determined the spherically symmetric structure of the Sun by using the optimally localized averaging techniques (e.g. Gough et al., 1996) to invert the mean frequencies of split mode multiplets. Figure 6 shows the relative difference between the square of the sound speed in the Sun and a reference solar model. The reference model was model $\mathrm{S}$ of Christensen-

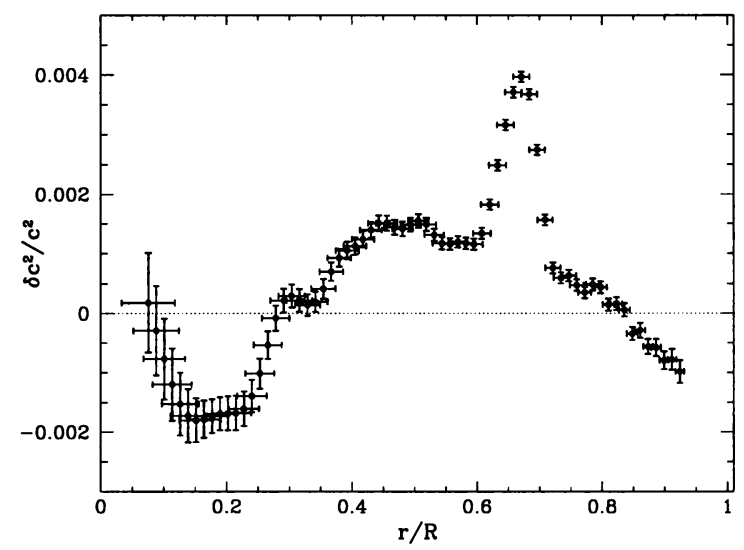

Figure 6. Relative differences between the squared sound speed in the Sun and a standard solar model as inferred from 2 months of the MDI data. The horizontal bars show the spatial resolution, and the vertical bars are error estimates.

Dalsgaard et al. (1996). This model was a standard evolutionary model computed using the most recent information on nuclear reaction rates (Bahcall and Pinsonneault, 1995) and radiative opacity and the equation of state (Rogers and Iglesias, 1996). The gravitational settling and diffusion of helium and heavier elements were taken into account following the theory by Michaud and Proffitt (1993).

The inversion results show that the maximum difference in the square of the sound speed between the model and the Sun is only $0.4 \%$. Two 
features of the sound-speed profile are particularly notable. The first is a narrow peak centered at $0.67 R$, just beneath the convection zone. This peak was previously detected in the LOWL (Basu et al., 1996) and GONG data (Gough et al., 1996) and is most likely due to a deficit of helium in this narrow region.

Another interesting feature is the sharp decrease of the sound speed compared to the model at the boundary of the energy-generating core, at $0.25 R$. It is quite possible that the drop in the sound speed results from an overabundance of helium at the edge of the solar core. The steep increase of the sound speed towards the solar center can be explained if helium is less abundant than in the standard solar model. This indicates that the helium abundance profile seems to be more flat in the solar core than it is in the standard solar model. Gough et al. (1996) came to a similar conclusion from the GONG data. However, if the transition at the edge of the core is really as sharp as we have found from the initial MDI data, then it strongly suggests material redistribution by macroscopic motion in the core, possibly induced by the instability of ${ }^{3} \mathrm{He}$ burning, as first suggested by Dilke and Gough (1972).

\section{Rotation Rate}

The internal rotation rate at three latitudes, 0 (equator), 30 and 60 degrees, is shown in Figure 7.

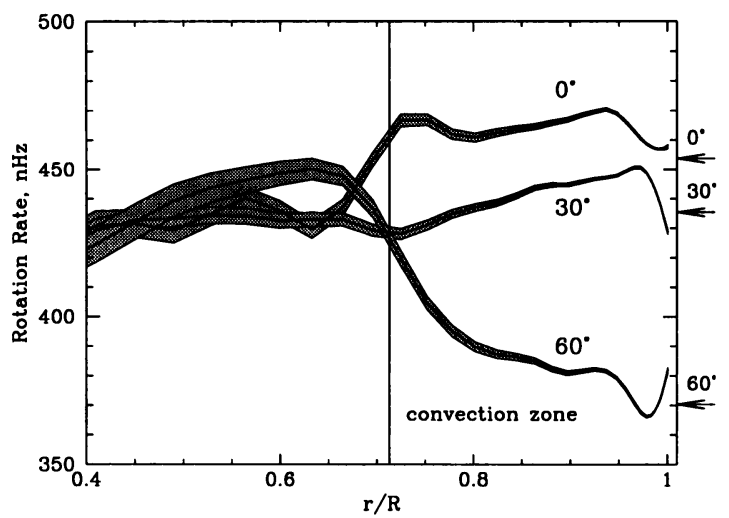

Figure 7. Solar rotation rate inferred from 2 months of MDI Medium-l data as a function of radius at three latitudes, $0^{\circ}, 30^{\circ}$, and $60^{\circ}$. The formal errors are indicated by the shaded regions. The arrows indicate the Doppler rotation rate directly measured on the surface.

The rotation is inferred using a regularized least-squares technique. The inversion results confirm the previous findings that latitudinal differential 
rotation occurs only in the convection zone, that the radiative interior rotates almost rigidly, and that there is a thin shear layer near the surface (e.g. Goode et al., 1991; Thompson et al., 1996). The most interesting is that the transition layer (tachocline) between the radiative and convection zone is mostly located in the radiative zone, at least at the equator where it is also fairly thin, certainly less than $0.1 R$. The layer seems to be wider at high latitudes.

The sharp radial gradient of the angular velocity beneath the convection zone gives strong evidence that the sharp narrow peak of the sound speed at $0.67 R$ seen in the structure inversion (Figure 6) is due to rotationallyinduced turbulent mixing in the tachocline (Spiegel and Zahn, 1992). The location and the width of the tachocline are also consistent with the requirement of recent dynamo theories of the solar cycle.

\section{Acknowledgments}

The authors acknowledge many years of effort by the engineering and support staff of the MDI development team at the Lockheed Palo Alto Research Laboratory (now Lockheed-Martin Advanced Technology Center) and the SOI development team at Stanford University. SOHO is a project of international cooperation between ESA and NASA. This research is supported by the SOI-MDI NASA contract NAG5-3077 at Stanford University.

\section{References}

Bahcall, J.N., and Pinsonneault, M.H. (1995) Rev. Mod. Phys., 67, 781.

Basu, S., Christensen-Dalsgaard, J., Schou, J., Thompson, M.J., and Tomczyk, S. (1996) Bull. Astr. Soc. India, 24, 147.

Christensen-Daalsgard, J., Däppen, W., and the GONG Team (1996) Science, 272, 1286.

Dilke, F.W.W., and Gough, D.O. (1972) Nature, 240, 262.

Duvall, T.L., Jr., Jefferies, S.M., Harvey, J.W., Osaki, Y., and Pomerantz, M.A. (1993) Astrophys. J., 410, 829.

Gabriel, M. (1992) Astron. Astrophys., 265, 771.

Goode, P.R., Dziembowski, W.A., Korzennik, S.G., and Rhodes, E.J. (1991) Astrophys. J., 367, 649.

Gough, D.O., Kosovichev, A.G., and the GONG Team (1996) Science, 272, 1296.

Kosovichev, A.G., Schou, J., Scherrer, P.H., and the MDI Team (1997) Solar Phys., in press.

Kumar, P., Fardal, M.A., Jefferies, S.M., Duvall, T.L., Jr., Harvey, J.W., and Pomerantz, M.A. (1994) Astrophys. J., 422, L29.

Michaud, G., and Proffitt, C.R. (1993) in A.Baglin and W.Weiss (eds), Inside the stars, ASP Conf. Series, vol. 40, San Francisco, p.246.

Nigam, R., and Kosovichev, A.G. (1996) in J. Provost and F.X. Schmider (eds), Sounding Solar and Stellar Interiors, Kluwer Acad. Publ., in press.

Rogers, F.J., and Iglesias, C.A. (1996) Astrophys. J., 456, 902.

Scherrer, P.H., and the MDI Team (1996) Solar Phys., 162, 129.

Schou, J. (1992) On the Analysis of Helioseismic Data, Thesis, Aarhus University .

Spiegel, E.A., and Zahn, J.-P. (1992) Astron. Astrophys., 265, 106.

Thompson, M.J., Toomre, J., and the GONG Team (1996) Science, 272, 1300. 